\title{
Sex hormone profiles and cellular changes of reproductive organs of mice experimentally infected with $C$. pseudotuberculosis and its exotoxin phospholipase D (PLD)
}

\author{
Zaid Khuder ${ }^{1}$, Abdinasir Yusuf Osman ${ }^{1}$, Faez Firdaus Jesse ${ }^{* 1}$, Abd Wahid \\ Haron $^{1}$, Abdul Aziz Saharee ${ }^{1}$, Jasni Sabri ${ }^{1}$, Rosnina Yusoff ${ }^{1}$ and Rasedee \\ Abdullah $^{1}$ \\ ${ }^{1}$ Faculty of Veterinary Medicine, Universiti Putra Malaysia, 43400 UPM Serdang, Selangor, Malaysia
}

\begin{abstract}
Caseous lymphadenitis has been globally a prevalent disease among farmed small ruminants for over a century. As its chronic disease, sex hormone profiles and cellular changes of reproductive organs in infections associated with Corynebacterium pseudotuberculosisis and its exotoxin phospholipase D have been very few or absent. This study was conducted to acquire a better way of understanding the reproductive pathophysiology response of Corynebacterium pseudotuberculosisis and its exotoxin in mouse model. In this study, 64 healthy mice, 2-3 weeks of old, were divided equally into 3 groups, where the first group of mice were interperitoneally inoculated with $1.0 \mathrm{ml}$ of sterile phosphate buffer solution $(P B S), p H 7$, the second group of mice were interperitoneally inoculated with $1.0 \mathrm{ml}$ of $10^{9}$ colony forming unit (CFU) of live $C$. pseudotuberculosis and the third group of mice were interperitoneally inoculated with $1.0 \mathrm{ml}$ of single dose of exotoxin (PLD) extracted from C. pseudotuberculosis. Following infection, clinical signs were observed and blood samples were collected by cardiac puncture for sex hormone analysis. For microscopic examinations, the mice were euthanized using cervical dislocation approach and the reproductive organs were collected. The results revealed that there was no significant differences $(p<0.05)$ in the concentration of estrogen and progesterone levels between whole cell and exotoxin groups. Concentration of progesterone in PLD treated group $(15.37 \pm 2.32 \mathrm{pg} / \mathrm{ml})$ was lower than control group $(17.61 \pm 2.91 \mathrm{pg} / \mathrm{ml})$. The concentration of testosterone in C. pseudotuberculosis $(2.98 \pm 3.70 \mathrm{pg} / \mathrm{ml})$ was significantly $(p<0.05)$ different from both control group $(16.58 \pm 3.67 \mathrm{pg} / \mathrm{ml})$ and PLD $(11.84 \pm 3.19 \mathrm{pg} / \mathrm{ml})$. Microscopically, cellular changes were observed in organs included ovaries, uterus, testicles and epididymis. In C. pseudotuberculosis infected group, infiltration of polymorph nuclear leukocytes with congestion, degeneration and necrosis were documented in almost all investigated parameters. For exotoxin PLD infected group, the observed cellular changes included severe hemorrhage, thrombus formation, degeneration, vacuolation and necrosis. Therefore, the results of this study indicated that there were significant differences in sex hormones and cellular changes of reproductive organs associated with infection Corynebacterium pseudotuberculosisis and its exotoxin phospholipase D (PLD).
\end{abstract}

Keywords: Caseous Lymphadenitis, C. pseudotuberculosis, Hormones, Phosholipase D, Reproduction

"Corresponding author. Mailing address: Department of Clinical Studies, Faculty of Veterinary Medicine, Universiti Putra Malaysia, 43400 Serdang, Selangor, Malaysia. Tel.: +60175550870. E-mail address: jesse@putra.upm.edu.my (Faez Firdaus Jesse).

\section{Introduction}

Caseous Lymphadenitis (CLA) is caused by Corynebacterium pseudotuberculosis, a gram positive, facultative anaerobic rod [1]. The disease is, most commonly, chronic in sheep and goats and it's characterized by encapsulated abscesses especially in superficial lymph nodes and as it can be disseminated deeper to visceral lymph nodes and organs [2]. The visceral form of CLA called Thin Ewe Syndrome is associated with low reproductive rates in sheep [3]. Other animal species in which infection with $C$. pseudotuberculosis is relatively common, include horses $[4,5,6]$, cattle [7, 8, 9], llamas and alpacas [10,11] and buffalo [12]. Although CLA could cause important economic losses associated with as loss of body condition and subsequent reproductive failure [13, 14], the virulence mechanisms of the agent have been scarcely characterized and the epidemiology of the infection is still poorly understood in many countries. CLA is transmitted via superficial skin cuts and abrasion especially during fighting and shearing process [15]. To date, there are only two virulence factors that have been identified in C. pseudotuberculosis; PLd and Mycloic acid [16]. PLD define as sphingomyelinspecific phospholipase, that catalysis sphingomyelin into phosphate and choline. Mycolic acid is a waxy coat on the outer surface of $C$. pseudotuberculosis. This waxy coat enables the organism to survive the harsh environmental conditions in the farm for long period and probably provides the microorganism with mechanical and biochemical protection against the hydrolytic enzymes in the macrophages lysosome [1, 16]. Both PLD and 
Sex hormone profiles and cellular changes of reproductive organs of mice experimentally infected mycolic acid play a major role in pathogenicity of CLA. The most susceptible organs for the infection include lungs and epididymis, followed by liver, spleen, retropharyngeal lymph nodes and udder [17]. Epididymis and udder were found to be most susceptible organs of CLA infections. In this, however, we assume that CLA may affect the fertility and subsequently the reproduction. [18] Examined rams and they found that CLA lesions frequently exist and lying adjacent to testes, spermatic cord and inguinal lymph nodes were enlarged, but $C$. pseudotuberculosis does not exist in the semen. CLA can cause loss of fertility and gradual emaciation [19]. Moreover, studies on CLA have been mentioned its negative effects on reproduction. However there is no currently study speculated the possible scenario of how CLA can affect the reproduction. Therefore, this study was carried out using mouse model to investigate what other researchers reported about CLA being insidious contributor to animal's infertility. The addressed question remains whether CLA possibly affects the hypopituitary gonadal axis (hormones) or affects the gonads itself resulting disturbance in reproduction.

\section{Materials and Methods}

Animals

Sixty four apparently healthy mice of both sexes, aged 8 weeks were used in this study. Mice were kept under standard condition for 2 weeks prior the experiment for acclimatization. The experiment was conducted according to the guide of the care and use of experimental animals, provided by Institutional Animal Care and Use Comity (IACUC). All experimental procedures were approved by Universiti Putra Malaysia and Animal Care Committee with reference No. (UPM/FPV/PS/3.2.1.551/AUP-R120).

\section{Corynebacterium pseudotuberculosis}

The bacterium was isolated from clinical case of CLA infections at UPM farm. The organism was biochemically identified. The inoculum was estimated at $1 \times 10^{9} \mathrm{CFU}$ using McFarland Standard.

Phospholipase D (PLD)

PLD was extracted using Zaki's method [20].

Experimental Design

The mice were divided into 3 groups; namely whole cell, exotoxin (PLD) and control groups. The infected groups (whole cell and exotoxin) consisted of 24 animals each where control group consisted of 16 animals. The control group was inoculated interperitoneally with $1 \mathrm{ml}$ of sterile PBS. The whole cell group was inoculated with $C$. pseudotuberculosis $1 \times 10^{9} \mathrm{CFU}$ where the exotoxin (PLD) group was challenged interpertoneally with $1 \mathrm{ml}$ of PLD. Blood was collected for hormone analysis and post mortem examination was performed on reproductive organs. The collected organs were processed for histo-pathological examination.

\section{Hormone Analysis}

Testosterone concentration was estimated by using an ELISA kit (No. 582701), Estrogen and Progesterone concentration was also estimated by using an ELISA kits (No. 582251 and No. 582601) respectively.

\section{Histopathology}

The harvested organs were placed in $10 \%$ buffered formalin for routine paraffin embedding and sectioning with subsequent staining using hematoxylin and eosin.

\section{Statistical Analysis}

Statistical analysis was performed using PASW Statistics 18, Release Version 18.0.0 (D3 SPSS, Inc., 2009, Chicago, IL). One way analysis of variance (ANOVA) was used with Duncan post hoc multiple comparisons.

\section{Hormones concentration}

\section{Results}

Testosterone levels in animals challenged with $C$. pseudotuberculosis were significantly different $(\mathrm{p}<0.05)$ from exotoxin (PLD) group where C. pseudotuberculosis infected group had lower concentrations compared to exotoxin group (Table 1). In contrast, progesterone concentration was not significantly different between the inoculated groups; yet the animals inoculated with exotoxin (PLD) showed lower concentration of progesterone hormone (Table 1). Estrogen hormone levels in infected groups were both significantly $(\mathrm{p}<0.05)$ different from those served as a control group.

\section{Histopathology}


Sex hormone profiles and cellular changes of reproductive organs of mice experimentally infected

Changes in the tissues were observed after 3 days post-inoculation in whole organism and after 4 hours in PLD challenged groups. However, the general changes in the groups inoculated with C. pseudotuberculosis were related to septicemia. These changes included congestion, infiltration of neutrophil and macrophages, degeneration, necrosis, hemorrhage and oedema. On the other hand, the exotoxin (PLD ) inoculated groups showed generalized toxemia where organs of these groups had congestion, oedema, thrombus formation and necrosis. The ovaries and the uterus in whole organism inoculated group showed congestion with infiltration of neutrophil and macrophages, oedema, degeneration and necrosis (Fig. 1\&3). In contrast, ovaries and uterus of exotoxin (PLD) inoculated animals showed severe congestion, profound thrombus formation and necrosis (Fig. 2\&4). Organs such as testis and epididymis showed congestion, oedema, infiltration of polymorphnuclear leukocytes, degeneration and necrosis in C. pseudotuberculosis inoculated animals (Fig. 5\&7). In exotoxin (PLD) inoculated animals testis and epididymis showed only severe congestion, thrombosis and necrosis (Fig. $6 \& 8)$.

Table 1 Sex hormone concentrations in mice following interperitoneally experimental infection with $C$. pseudotuberculosis and exotoxin (PLD) on (Mean \pm SE)

\begin{tabular}{lccc}
\hline & \multicolumn{3}{c}{ Groups } \\
\hline Hormones $(\mathrm{pg} / \mathrm{ml})$ & \multicolumn{1}{c}{ Control } & C. pseudotuberculosis & Exotoxin (PLD) \\
\hline Estrogen & $29.66 \pm 4.32$ & $41.53 \pm 3.55$ & $34.43 \pm 2.60$ \\
Progesterone & $17.61 \pm 2.91$ & $23.19 \pm 2.11$ & $15.37 \pm 2.32$ \\
Testosterone & $16.58 \pm 3.67$ & $2.98 \pm 3.70$ & $11.84 \pm 3.19$ \\
\hline
\end{tabular}

*Significant value $\mathrm{p}<0.05$. Comparison between challenged groups and control group mice



Fig. 1: Ovary of mouse challenged with $C$. pseudotuberculosis: Infiltration of polymorph nuclear leukocytes into the lumen of ovulated follicle (HE, X400).

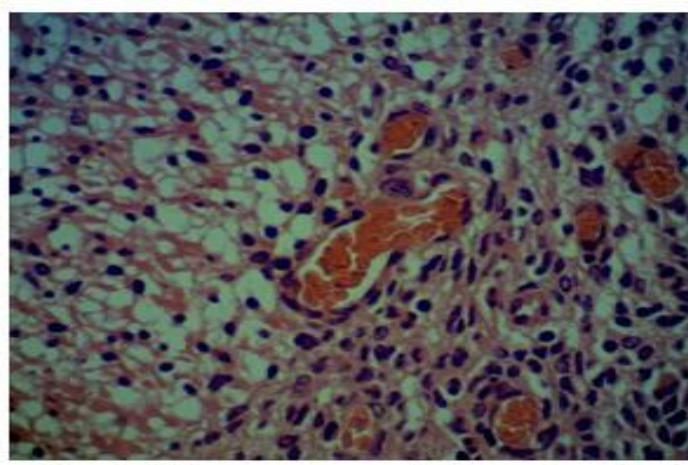

Fig. 3: Uterus mouse challenged with $C$ pseudotuberculosis: sever congestion, infiltration of neutrophil, macrophages, degeneration and necrosis of muscular layer $(\mathrm{HE}, \mathrm{X} 400)$.



Fig. 2: Ovary of mouse inoculated with exotoxin (PLD): Generalized congestion, sever thrombosis, degeneration and necrosis stromal cells (HE, X400).



Fig. 4: Uterus of mouse inoculated with exotoxin (PLD): generalized congestion, thrombosis, hyaline degeneration and muscle cell necrosis ( $\mathrm{HE}, \mathrm{X} 400)$. 


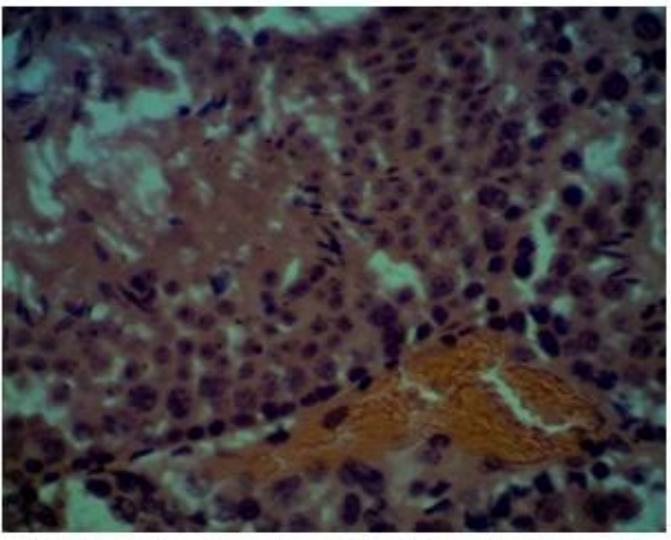

Fig. 5: Testes showing Infiltration of polymorph nuclear leukocytes, Congestion, degeneration and necrosis of spermatogonia in mouse challenged with C. pseudotuberculosis ( $\mathrm{HE}, \mathrm{X} 400)$.

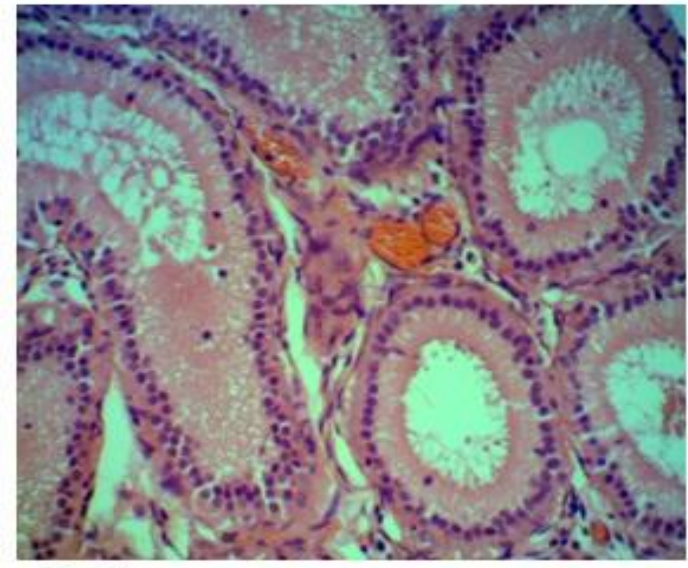

Fig. 7: Epididymus of mouse challenged with C. pseudotuberculosis: Infiltration of neutrophil, macrophages, venous congestion, degeneration and necrosis of tubular epithelia $(\mathrm{HE}, \mathrm{X} 200)$.

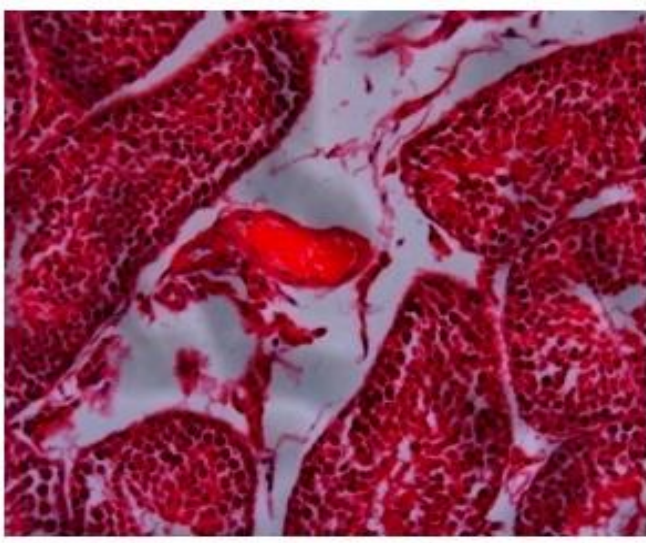

Fig. 6: Testes showing generalized congestion, profound edema, thrombus inside the vein and necrosis of some spermatogonia in mouse challenged with exotoxin (PLD), (HE, X200).

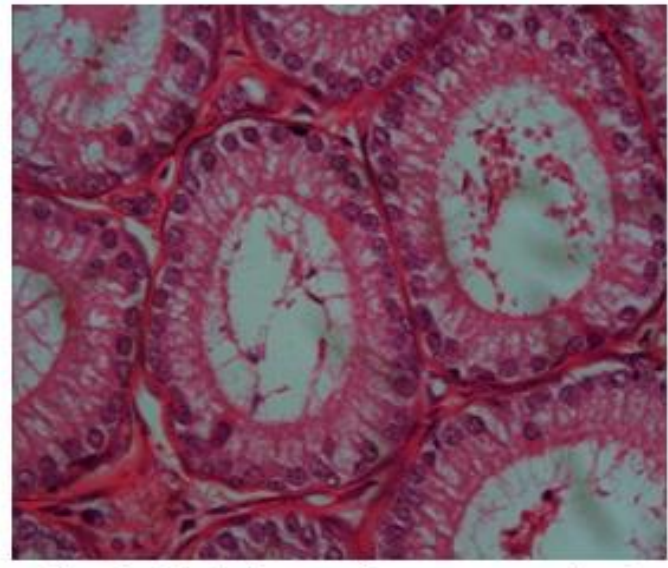

Fig. 8: Epididymus of mouse inoculated with exotoxin (PLD): generalized congestion, hemorrhage inside the lumen of epididymus tubules and necrosis of the epithelial cells of tubules (HE, X200).

\section{Discussion}

The insidious effects of CLA on reproduction often make the veterinarians and farmers overlooked its direct relation to fertility reduction and this contributes to the fact that farmers have little concern about CLA. This study for the first time reports the effects of CLA on reproductive hormones concentration and histopathological changes of reproductive organs in mouse model.

Blood collection for hormone analysis and postmortem performances on reproductive organs enabled documentation of observations and comparison of hormones concentration between the animals inoculated with C. pseudotuberculosis and those challenged with exotoxin (PLD). Microscopic lesions were observed 3 days after inoculation of whole organism and after $4 \mathrm{~h}$ of PLD inoculation. Although many studies have mentioned that chronic diseases such as CLA and John's disease could affect the reproduction of farm animals [21], yet these studies did not explain the mechanism of how these diseases disrupt or interrupt the reproduction system. Our findings showed that both $C$. pseudotuberculosis and exotoxin (PLD) have affected the sex hormone concentration, especially testosterone and in less extent progesterone. The tissue damages that have been observed in testis and ovaries may contribute to the low hormone concentration, in which the hormone producing cells have been affected or the hypo-pituitogonadal axis has been interrupted. Reproduction is a complex process that requires many factors to be achieved. Synergy of hypothalamus releasing hormones and pituitary tropic hormones are crucial for production of gonadal hormones (testosterone, estrogen, progesterone) to maintain the cyclicity of animal's reproduction. Furthermore any internal or external interruption of such precise process can lead to low fertility or sterility and ensue in failure of reproduction. [22] Found that exotoxin produced by Corynebacterium diptheriae has the capacity to inhibit protein synthesis in mammalian cells and causing death. Exotoxin (PLD) has the ability to catalyze the sphingomyelin [23]. The biological activities of exotxin PLD could explain the low hormone concentration by two mechanisms; direct action of PLD on 
Sex hormone profiles and cellular changes of reproductive organs of mice experimentally infected hormone producing cells compromising the function of cell membrane and cause cell death and the inhibition protein synthesis which means in other words inhibition of enzymes that are highly necessary for hormone production. [24] Described in detail the histopathological lesions in mice inoculated with C. pseudotuberculosis of general septicemic lesions such as generalized congestion, infiltration of nuetrophils and macrophages, hemorrhage, degeneration and necrosis. In this study the lesions observed in reproductive organs tissues were similar to those reported by [23] in almost all investigated parameters. [21] Reported that CLA can affect reproductive health indirectly through reduction of appetite, production of fever, loss of weight and loss of ambulation. CLA lesions can be found in internal organs as well as udder, and less common in testis, scrotum and uterus [25]. However, our experimental study using mouse model reports for the first time that changes in reproductive might occur due to direct action of $C$. pseudotuberculosis on reproductive tissues or indirectly by host tissue reaction to the pathogen in which the immune system take place.

As mentioned earlier, exotoxin (PL) has several biological activities such as dermonecrosis [26], lethality [27], interfering with ovine neutrophil chemotaxis and it is lethal to neutrophil itself [28]. In our study, mice inoculated with PLD showed severe histopathological changes in testis, epididymis, ovaries which were associated with toxemic manner such as severe generalized congestion, edema, hemorrhage, degeneration, sever thrombosis and necrosis. PLD can cause increase in vascular endothelial membrane permeability as a result of hydrolysis of sphingomyelin and leakage of plasma proteins into surrounding tissues [29]. However, this could explain the oedema found in tissues after inoculation of PLD. Furthermore, Exotoxin (PLD) may cause complete destruction of capillaries wall that consist of single layer of endothelial cells leading to hemorrhage and/or thrombosis as it revealed by histopathological examination. It's well known that PLD has lethal effect on cells [27], this cytotoxic effect may explain the necrosis appeared upon examination of tissues from PLD inoculated mice.

\section{Conclusion}

Finally, our study of experimental model highlighted the impact of CLA on reproduction for both sexes. Knowledge of such effects would increase our understanding of CLA on reproductive system. It would also further enhance the level of awareness regarding to this devastating disease to both reproduction and reproductivity.

\section{Acknowledgements}

The authors are grateful to Mr. Yap Keng Chee and Mr. Mohd Jefri Bin Norsidin for their assistance in preparation and photography of histological sections. This work was funded under the Research University Grant Scheme (RUGS), Universiti Putra Malaysia.

\section{References}

[1] Williamson, LH. Caseous lymphadenitis in small ruminants. Veterinary Clinics of North America: Food Animal Practice, 17, 2001, 359-371.

[2] Stoops, SG., Renshaw, HW, and Thilsted, JP. Ovine caseous lymphadenitis: disease prevalence, lesion distribution, and thoracic manifestation in a population of mature culled sheep from Western United States. American Journal of Veterinary Research, 45 , 1984, 557-561.

[3] Gates, NL., Everson, DO, and Hulet, CV. Effects of thin ewe syndrome on reproductive efficiency. Journal of the American Veterinary Medical Association, 171, 1977, 1266-1267.

[4] Addo, P.B., Wilcox, G.E, and Taussig, R. Mastitis in a mare caused by Corynebacterium ovis. Veterinary Recearch, 95, $1974,193$.

[5] Miers, KC, and Ley, WB. Corynebacterium pseudotuberculosis infection in the horse: study of 117 clinical cases and consideration of etiopathogenesis. American Journal of Veterinary Medical Association, 177, 1980, 250-253.

[6] Poonacha, K.B, and Donahue, J.M. Abortion in a mare associated with Corynebacterium pseudotuberculosis infection. Journal of Veterinary Diagnostic Investigation, 7, 1995, 563-564.

[7] Adekeye, J.D., Shannon, D, and Addo, P.B. Mastitis in a cow caused by Corynebacterium pseudotuberculosis (C. ovis). Veterinary Recearch, 106, 1980, 270.

[8] Anderson, M.L., Lean, I. J, and Blanchard, P.C. Corynebacterium pseudotuberculosis associated skin disease of Holstein cattle in the San Joaquin Valley, California. Bovine Practice, 25, 1990, 73-75.

[9] Yeruham, I., Elad, D., Van Ham, M., Shpigel, N.Y, and Perl, S. Corynebacterium pseudotuberculosis infection in Israeli cattle: clinical and epidemiological studies. Veterinary Record, 140, 1997, 423-427.

[10] Braga,W.U., Chavera, A., and Gonzalez, A. Corynebacterium pseudotuberculosis infection in highland alpacas (Lama pacos) in Peru. Veterinary Record, 159, 2006, 23-24.

[11] Braga,W., Schul, S., Nũnez, A., Pezo, D, and Franco, E. A primary Corynebacterium pseudotuberculosis lowdose infection in alpacas (Lama pacos) protects against a lethal challenge exposure. Small Ruminanr Research, 72, 2007, 81-86.

[12] Ali, H.S., and Zaitoun, A.M. Studies on cutaneous suppurative lymphangitis in buffaloes at Assiut Governorate-Egypt. Assiut Veterinary Medical Journal, 41, 1999, 208-222.

[13] Dorella, F.A., Pacheco, L.G., Oliveira, S.C., Miyoshi, A, and Azevedo, V. Corynebacterium pseudotuberculosis: microbiology, biochemical properties, pathogenesis and molecular studies of virulence. Veterinary Research, 37, 2006, 201-218.

[14] Kuria, JKN., Mbuthia, PG., Kang'ethe, EK, and Wahome, RG. Caseous Lymphadenitis in Goats: The Pathogenesis, Incubation Period and Serological Response after Experimental Infection. Veterinary Research Communication, 25, 2001, 89-97.

[15] Batey, R.G. Pathogenesis of caseous lymphadenitis in sheep and goats. Australian Veterinary Journal, 63, $1986,269-273$.

[16] Baird, GJ, and Fountaine, MC. Corynebacterium pseudotuberculosis and its role in ovine caseous lymphadenitis. Journal of Comparative Pathology, 137, 2007, 179-210. 
[17] Unanian, MM., Felicianosilva, AED, and Pant, KP. Abscesses and caseous lymphadenitis in goats in tropical Semi-Arid north-east Brazil. Tropical Animal Health and Production, 17, 1985, 57-62.

[18] Williamson, P, and Nairn, ME. Lesion caused by Corynebacterium pseudotuberculosis in the scrotum of rams. Australian Veterinary Journal, 56, 1980, 496.

[19] Connner, KH., Quirie, MM., Baird, G, and Donachie, W. Characterization of United Kingdome isolates of Corynebacterium pseudotuberculosis using pulsed field gel electrophoresis. Journal of Clinical Microbiology, 38, 2000, 2633-2637.

[20] Zaki, M. M. The application of a new technique for diagnosing Corynebacterium ovis infection. Research inVeterinary Science, 9 , 1968, 489-493.

[21] Peterhans, E., Greenland, T, and Badiola, J. Routes of transmission and consequences of small ruminant lentiviruses (SRLV's) infection and eradication schemes. Veterinary Research, 35, 2004, 257-274.

[22] Sivananda, N., Ajesh, S., Manju, C., Sugapriya, P., Mukund Sudharshan MG., Maheswari, G., Priscilla, K., Palaniappan, C, and Sekar, B. Cytotoxic effect of diphtheria toxin in mammalian cell lines. Indian Journal of Science and Technology, 3, 2010, $1177-$ 1179.

[23] Pepin, M., Paton, M, and Hodgson, AL. Pathogenesis and epidemiology of Corynebacterium pseudotuberculosis infection in sheep. Current Topics in Veterinary Research, 1, 1994, 63-82

[24] Jesse, FFA., Sang, SL., Saharee, AA, and Shahirudin, S. Pathological Changes in the Organs of Mice Model Inoculated with Corynebacterium pseudotuberculosis Organism. Pertanika Journal of Tropical and Agricultural Science, 34, 2011, 145 - 149.

[25] Valli,V.E.O, and Parry, B.W. Caseous lymphadenitis, In: Pathology of Domestic Animals, Vol. 3, 4th Edit., K.V.F. Jubb, P.C. Kennedy and N. Palmer, Eds, Academic Press, San Diego, pp., 1993, 238-240.

[26] Muckle, CA, and Gyles, CL. Exotoxic activities of Corynebacterium pseudotuberculosis. Current Microbiology, 13, 1986, 57-60.

[27] Brogden, K.A, and Engen, RL. Alterations in the phospholipid composition and morphology of ovine erthrocytes after intravenou s inoculation of Corynebacterium pseudotuberculosis. American Journal of Veterinary Research, 51, 1990, 874-877.

[28] Yozwiak, ML, and Songer, JG. Effect of Corynebacterium pseudotuberculosis phospholipase D on viability and chemotactic responses of ovine neutrophils. American Journal of Veterinary Research, 54, 1993, 392-397.

[29] Carne, HR, and Onon, EO. Action of Corynebacterium ovis exotoxin on the endothelial cells of blood vessels. Nature, 271, 1978 ,246-248. 